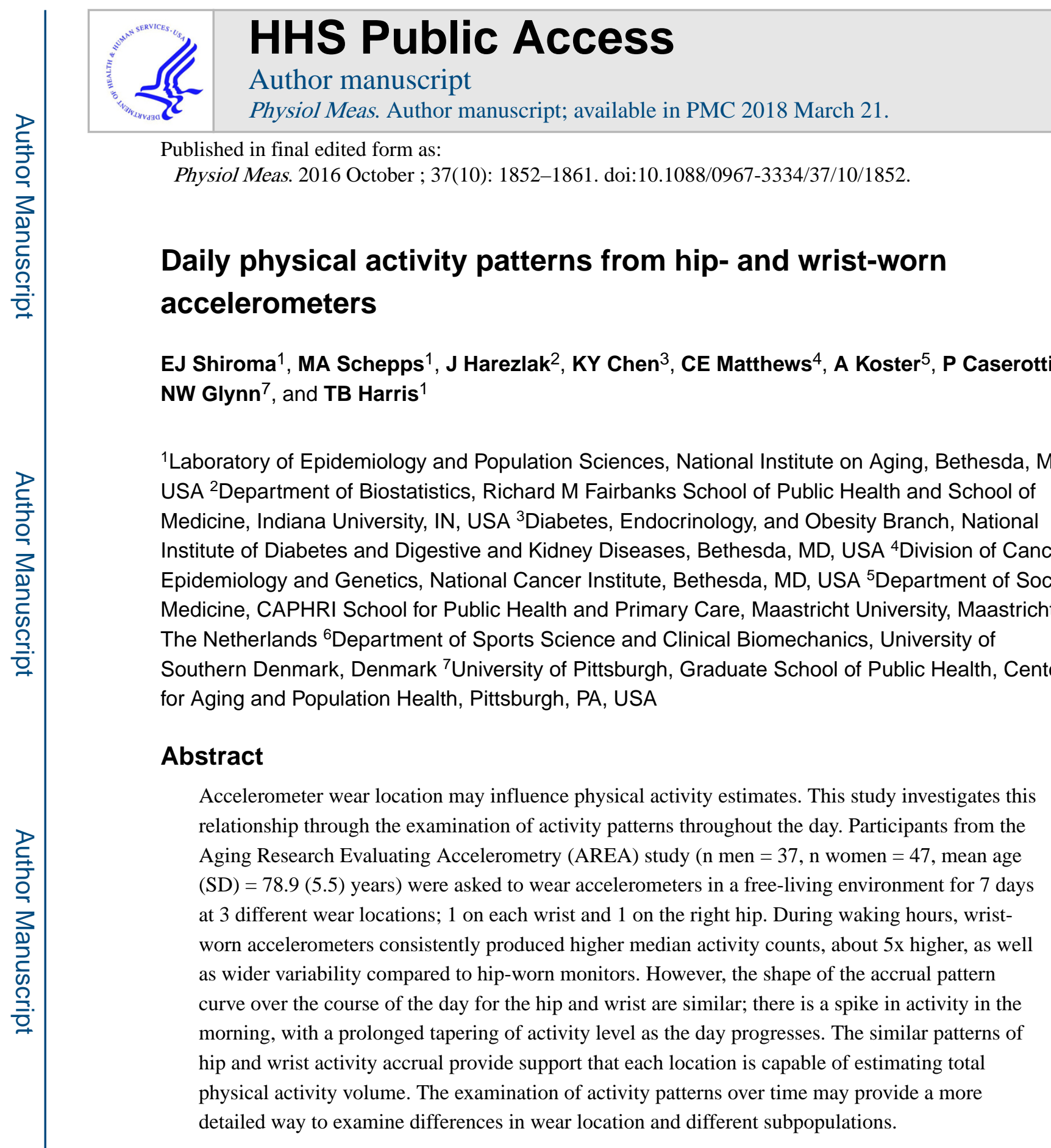

Keywords

Physical activity; epidemiology; exercise; accelerometer; patterns; aging

Corresponding Author: Eric J Shiroma, ScD, MEd, 7201 Wisconsin Ave, Gateway Bldg, Suite 3C309, Bethesda, MD 20814, P: 301-496-1178, F: 301-496-4006, Eric.shiroma@nih.gov.

Conflict of Interest

The authors have no conflict of interest. 


\section{Introduction}

Accelerometers are an increasingly utilized physical activity assessment tool and can be worn at a variety of locations on the body (Troiano et al., 2014, Lee and Shiroma, 2014). However, accelerometers worn at different locations on the body provide different information regarding physical activity, complicating comparisons across studies (Cleland et al., 2013, Swartz et al., 2000, Troiano et al., 2014). One of the main challenges when comparing monitor locations is that accelerometers assess movement, not behavior, using a metric called activity counts. The same behavior (e.g. playing tennis) will result in different movement from different parts of the body and thus the monitors at each location register different counts. Cutpoints have been developed to characterize these movements and counts into intensity levels for hip-worn monitors (Freedson et al., 1998, Sasaki et al., 2011, Matthew, 2005), but there are currently no widely used cutpoints for the wrist-worn accelerometers. Thus, one of the only metrics available to compare hip and wrist monitors is the total number of counts, with the known limitation that the same activity count derived from various locations may not represent the same movement or behavior.

As accelerometers are able to assess movements in real-time, one of the greatest strengths of an accelerometer is the incorporation of time to assess one important attribute of physical activity - when and how frequently does an activity occur. Previously, accelerometers have been used to increase precision of the duration of activity and conduct bout analyses (Shiroma et al., 2013, Jefferis et al., 2015, Hooker et al., 2015, Tucker et al., 2011). However, patterns throughout the day may be equally useful (Schrack et al., 2014, Sartini et al., 2015, Martin et al., 2014, Arnardottir et al., 2013). In this case, examining patterns over time allows for a comparison across wear locations without a direct translation of counts to intensity for each location. This study uses accelerometer activity patterns throughout the day to examine the similarities and differences in physical activity estimates from hip and wrist locations among older men and women in a free-living environment.

\section{Methods}

\section{Study Population}

The Aging Research Evaluating Accelerometry (AREA) study, part of the Developmental Epidemiologic Cohort Study (DECOS) (Lange-Maia et al., 2015), is a methodological study designed to examine the impact of accelerometer wear location on assessment of physical activity and sedentary behavior among 89 older adults. Detailed study protocols are described in detail elsewhere (Lange-Maia et al., 2015) Briefly, participants (aged 70 years and older) without self-reported health contraindications to fitness testing (cancer, lung disease, cardiovascular disease, knee or hip replacement, or spinal surgery) and an inability to perform basic mobility tasks (i.e., severe pain, aching, or stiffness while walking) were recruited and enrolled in the study. During the free-living component of the AREA study (2012-2013), participants were asked to simultaneously wear 3 ActiGraph GT3X+ accelerometers: 1 on each wrist and 1 above the right hip bone. Of the 89 eligible participants, 4 were excluded due to missing data or device failures, and 1 participant was excluded for insufficient wear time (described below). This resulted in a final analysis sample of 84 participants. The study was approved by the Institutional Review Boards of the 
University of Pittsburgh and National Institute on Aging; all participants provided written informed consent.

\section{Accelerometer Measures}

Participants were instructed to wear the accelerometers (time-synchronized at the device deployment) for 7 days; hip monitor during waking hours and wrist monitors continuously. Hip and wrist accelerometer data, using all three axes, were screened for periods of wear ("wear time") using methods described by Choi et al (Choi et al., 2011). Briefly, non-wear time was defined as 90 consecutive minutes of zero counts, with an allowance of 2-minutes of nonzero counts provided there were 30-minute consecutive zero count windows up and downstream. A valid wear day consisted of at least 10 hours of wear time. Participants were required to have at least 1 valid day to be included in the sample. In order to compare accelerometer data across wear locations, we limited the data from all devices to times when the two wrist- and hip-worn devices were worn. Data analyses were restricted to valid data within an a priori "waking day" of 05:00 to 23:00.

Accelerometer-assessed physical activity was defined as the weekly average vector magnitude (VM) counts per minute (cpm), a metric incorporating movement across all three axes of the accelerometer and wear time. VM cpm was summarized for 2 sets of analyses: 1) the hour and 2) the time of day (morning (05:00 to 09:59); midday (10:00 to 13:59); afternoon (14:00 to 17:59); and evening (18:00 to 22:59)). Aggregation of data by hour was used to reduce the number of statistical tests and to group times into more anecdotal behavior time frames.

\section{Statistical Analyses}

VM cpm patterns were examined visually by plotting the median (interquartile range) of the hourly activity levels for each wear location and gender. VM cpm were transformed using a natural $\log$ transformation for statistical testing due to skewness on the original scale. Mean (SD) differences in absolute VM cpm levels between hip and wrist locations for each time of day (morning, midday, afternoon, evening) were tested using pairwise t-tests. Time of day analyses were conducted separately for each time of day and gender.

To examine relative activity accumulation rates, a cumulative distribution plot was constructed for each location and gender. We then calculated the mean (SD) time by which $25 \%, 50 \%$, and $75 \%$ of the day's total activity had been completed. Differences in the time since the "beginning of waking day" were estimated for each location pair (hip and dominant wrist, hip and nondominant wrist, and between wrists) using pairwise t-tests. The "beginning of waking day" was defined as the time when the first valid minute of accelerometer data occurred after 05:00 each day.

\section{Results}

On average, participants $(\mathrm{n}$ men $=37, \mathrm{n}$ women $=47)$ were $78.9(\mathrm{SD}=5.5)$ years old, had a body mass index of $26.5(3.8) \mathrm{kg} / \mathrm{m}^{2}$, and were predominantly right-handed (95.2\%). Men and women did not differ significantly in terms of age, body mass index, or handedness. 
In general, the median hourly VM cpm level from the dominant wrist is higher than the nondominant wrist (Figure 1). The interquartile ranges from the dominant and nondominant wrist overlap greatly for each hour of the day. These trends between dominant and nondominant wrists are similar for both men and women. When comparing the hip and wrist, absolute wrist VM cpm are generally 5 times higher than hip counts during periods of activity (Figure 2). However, the shape of the curve over the course of the day for the hip and wrist are similar; there is a spike in VM cpm early in the day, with a prolonged tapering as the day progresses. Men and women have a similar trend in VM cpm throughout the day for both the hip and the wrist.

The mean VM cpm, on the log-transformed scale, is lower in hip compared to the wrists at each time of day and for both men and women $(\mathrm{p}<0.001$, Table 1). Dominant and nondominant wrists produced statistically different VM cpm estimates $(\mathrm{p}<0.05)$, with the one exception of mornings for women $(\mathrm{p}=0.46)$. However, the absolute differences appear to be very small. Overall, men and women appear to have very similar VM cpm at each point throughout the day (Table 1).

Relative accumulation rates using a cumulative distribution plot allow for a simple adjustment of the markedly different absolute activity counts from the hip-worn and wristworn monitors. The cumulative distribution plot shows that both men and women have similar trends in relative activity accrual throughout the course of the day independent of monitor wear location (Figure 3). Examining this more closely and quantitatively (Table 2), the mean difference in time since waking to reach $25 \%, 50 \%$, and $75 \%$ of the daily VM total is not statistically different between dominant and nondominant wrists $(p>0.05)$ in both men and women, except for women at $75 \%(\mathrm{p}=0.04)$. The mean difference in time between dominant and nondominant wrist ranges from 0.1 to 0.2 hours.

The time to reach $25 \%$ of the daily VM total is greater in the hip compared to the nondominant wrist in both men and women, however it is only statistically significant among women $(\mathrm{p}$ women $=0.04 ; \mathrm{p}$ men $=0.12$; Table 2 ). However as the day progresses, the hip reaches $50 \%$ and $75 \%$ earlier than the wrist. This is statistically significant for both $50 \%$ and $75 \%$ in men, but only $75 \%$ in women, although the absolute difference is similar $(0.4$ hours in men and 0.3 hours in women). As the cumulative plot must reach $100 \%$, and the hip reached $75 \%$ faster than the wrist, the wrist monitors must detect more activity at the end of the day in both men and women.

\section{Discussion}

This study examines accelerometer-assessed physical activity from the hip and wrist among older adults in a free-living environment. One of the main difficulties in comparing wear locations and different devices is that as accelerometers measure movement not behavior, the output of the monitor will vary based on location even during the same behavior. We proposed incorporating time, one of the accelerometer's greatest strengths, in order to help bridge this limitation, using a combination of time and VM to examine patterns of activity throughout the day. By examining how and when physical activity is accrued throughout the day, we can get a sense of an underlying dimension of activity independent of device or 
location. For example, the cumulative distribution plot allows us to examine the relative accrual of activity at each wear location without the need to directly equate the counts. When examined using the patterns of activity accumulation over the day, we observed the hip and wrist monitors appear to assess total activity volume similarly.

\section{Accelerometer Wear Locations}

Accelerometer studies have used several different wear locations as each location has its strengths and weaknesses. Two of the most popular locations are the hip and wrist. Hip-worn monitors, often seen as the 'traditional' placement for adult studies, are believed to best detect full body movement at or close to the center of mass with minimal participant burden and have been employed in several large surveillance studies including the National Health and Nutritional Examination Survey (NHANES) (Troiano et al., 2008, Shiroma et al., 2013, Howard et al., 2015, Arnardottir et al., 2013, Peters et al., 2010, Jefferis et al., 2015). However, the hip has been noted to be uncomfortable, particularly when participant were sleeping (Troiano et al., 2014). Wrist monitors are increasingly being used due to the ease of wear and high levels of adherence, particularly among younger participants (Rowlands et al., 2014, Troiano et al., 2014). It remains unknown if wrist monitors are able to detect locomotion or posture as well as the hip ones. Comparing studies with monitors worn at various locations may be problematic if body locations impact physical activity assessment.

In this study, we observed markedly higher counts $(\sim 5 \mathrm{x})$ when using the nondominant wrist monitor compared to the hip monitor, confirming other studies' previous findings (Hildebrand et al., 2014, Trost et al., 2014, Rowlands et al., 2014, Schaefer et al., 2014, Zhang et al., 2012, Tudor-Locke et al., 2015). As dominant wrist activity counts are generally higher than nondominant wrist activity counts, it would be expected that the difference between hip and wrist would be larger when examining the dominant wrist. However, when activity is plotted by time, the shapes of the curves look similar independent of wear location. We observed an initial spike in activity early in the day with a prolonged taper throughout the day in both the hip and wrist monitors. Similar patterns have been seen in other studies examining activity levels throughout the day at both hip and chest locations (Schrack et al., 2014, Sartini et al., 2015, Martin et al., 2014, Arnardottir et al., 2013).

However, this does not imply that the hip and wrists are engaged in the same behavior during all of this time. In fact, activity pattern analyses may shed light on when the difference in activity detection is not simply an artifact of the location but an actual difference in behavior. We observe that hip-worn devices detect more activity during the middle of the day, perhaps reflecting more ambulatory activity, whereas wrist-worn devices detect more activity later in the day. It remains unknown if this difference is behavioral in nature or what implications this has in terms of energy expenditure or health. Hip-worn devices may be capturing whole body movement, whereas wrist-worn devices may be capturing most whole body movement in addition to wrist or upper-body specific movements. Framing activity with a reference around time of day may help in identifying behavior patterns using more advanced statistical techniques such as machine learning. Further research is needed to examine these patterns from other wear locations such as the thigh or ankle as well as other types of accelerometers. 


\title{
Gender Differences
}

While the aim of this study was not to directly compare men and women in terms of activity patterns, we were able to describe patterns separately by gender. We observed, on average, men and women have very similar activity levels throughout the day. However, differences by gender were more striking for the wrist than the hip. One could hypothesize that from these data that men and women are participating in similar whole body movements as would be detected by hip monitors, but different amounts of upper-body activities such as household activities such as cleaning, meal preparation, and childcare. As discussed above, more research is needed to examine how these locations may reflect the underlying behavioral differences.

\section{Strengths \& Limitations}

To our knowledge, this study is the first to incorporate patterns of activity throughout the day to examine differences in VM output across wear locations. Furthermore, this study was conducted in a free-living environment in a population of older men and women, providing comparisons between the genders in the understudied older population. Several limitations are worth discussing. This analysis does not include any "gold standard" energy expenditure or activity measure such as $\mathrm{VO}_{2}$ assessment or direct observation. We were unable to know the type of activity, intensity, or duration from the monitor data. Thus, it is not possible to evaluate hip and wrist assessments in terms of validity relative to a known standard. In addition, AREA participants may be healthier than other similarly-aged populations due to exclusion criteria of no prevalent cancer, cardiovascular or lung disease, or conditions counter-indicating fitness testing. This may limit generalizability of the findings as it is unknown how these conditions may alter daily activity patterns.

\section{Conclusions}

While hip and wrist monitors produce different absolute VM activity values, the consistency of the patterns of hip and wrist activity accrual throughout the day provide support for the assessment of a similar underlying physical activity dimension, independent of wear location. Combining time information with accelerometer data may provide a more detailed way to examine differences in wear location and across subpopulations.

\section{Acknowledgments}

This study was supported by the Intramural Research Program of the National Institutes of Health, National Institute on Aging and by the research grants AG024826, AG024827, AG036594, and AG000181 from the National Institutes of Health. A Koster has received funding from the European Union Seventh Framework Programme (FP7-PEOPLE-2011-CIG) under grant agreement PCIG09-GA-2011-293621. NW Glynn was supported in part by National Institute on Aging Professional Services Contract HHSN271201 100605P. We are grateful to the staff of the AREA and DECO studies, particularly BS Lange-Maia, ME Garcia, and JR Treinish.

\section{Abbreviations}

\author{
AREA Aging Research Evaluating Accelerometry \\ DECOS Developmental Epidemiologic Cohort Study \\ CPM Counts Per Minute
}


VM Vector Magnitude

\section{References}

Arnardottir NY, Koster A, Van Domelen DR, Brychta RJ, Caserotti P, Eiriksdottir G, Sverrisdottir JE, Launer LJ, Gudnason V, Johannsson E, et al. Objective measurements of daily physical activity patterns and sedentary behaviour in older adults: Age, Gene/Environment Susceptibility-Reykjavik Study. Age Ageing. 2013; 42:222-9. [PubMed: 23117467]

Choi L, Liu Z, Matthews CE, Buchowski MS. Validation of accelerometer wear and nonwear time classification algorithm. Med Sci Sports Exerc. 2011; 43:357-64. [PubMed: 20581716]

Cleland I, Kikhia B, Nugent C, Boytsov A, Hallberg J, Synnes K, Mcclean S, Finlay D. Optimal placement of accelerometers for the detection of everyday activities. Sensors. 2013; 13:9183-200. [PubMed: 23867744]

Freedson PS, Melanson E, Sirard J. Calibration of the Computer Science and Applications, Inc. accelerometer. Med Sci Sports Exerc. 1998; 30:777-81. [PubMed: 9588623]

Hildebrand M, Vt VaNH, Hansen BH, Ekelund U. Age group comparability of raw accelerometer output from wrist- and hip-worn monitors. Med Sci Sports Exerc. 2014; 46:1816-24. [PubMed: 24887173]

Hooker SP, Hutto B, Zhu W, Blair SN, Colabianchi N, Vena JE, Rhodes D, Howard VJ. Accelerometer measured sedentary behavior and physical activity in white and black adults: The REGARDS study. J Sci Med Sport. 2015

Howard VJ, Rhodes JD, Mosher A, Hutto B, Stewart MS, Colabianchi N, Vena JE, Blair SN, Hooker SP. Obtaining Accelerometer Data in aN ational Cohort of Black and White Adults. Med Sci Sports Exerc. 2015; 47:1531-7. [PubMed: 25333247]

Jefferis BJ, Sartini C, Shiroma E, Whincup PH, Wannamethee SG, Lee IM. Duration and breaks in sedentary behaviour: accelerometer data from 1566 community-dwelling older men (British Regional Heart Study). Br J Sports Med. 2015; 49:1591-4. [PubMed: 25232029]

Lange-Maia BS, Newman AB, Strotmeyer ES, Harris TB, Caserotti P, Glynn NW. Performance on fast- and usual-paced 400-m walk tests in older adults: are they comparable? Aging Clin Exp Res. 2015; 27:309-14. [PubMed: 25373610]

Lee IM, Shiroma EJ. Using accelerometers to measure physical activity in large-scale epidemiological studies: issues and challenges. Br J Sports Med. 2014; 48:197-201. [PubMed: 24297837]

Martin KR, Koster A, Murphy RA, Van Domelen DR, Hung MY, Brychta RJ, Chen KY, Harris TB. Changes in daily activity patterns with age in U.S. men and women: National Health and Nutrition Examination Survey 2003-04 and 2005-06. J Am Geriatr Soc. 2014; 62:1263-71. [PubMed: 24962323]

Matthew CE. Calibration of accelerometer output for adults. Med Sci Sports Exerc. 2005; 37:S512-22. [PubMed: 16294114]

Peters TM, Moore SC, Xiang YB, Yang G, Shu XO, Ekelund U, Ji BT, Tan YT, Liu Da K, Schatzkin A, et al. Accelerometer-measured physical activity in Chinese adults. Am J Prev Med. 2010; 38:583-91. [PubMed: 20494234]

Rowlands AV, Olds TS, Hillsdon M, Pulsford R, Hurst TL, Eston RG, Gomersall SR, Johnston K, Langford J. Assessing sedentary behavior with the GENEActiv: introducing the sedentary sphere. Med Sci Sports Exerc. 2014; 46:1235-47. [PubMed: 24263980]

Sartini C, Wannamethee SG, Iliffe S, Morris RW, Ash S, Lennon L, Whincup PH, Jefferis BJ. Diurnal patterns of objectively measured physical activity and sedentary behaviour in older men. BMC Public Health. 2015; 15:609. [PubMed: 26141209]

Sasaki JE, John D, Freedson PS. Validation and comparison of ActiGraph activity monitors. J Sci Med Sport. 2011; 14:411-6. [PubMed: 21616714]

Schaefer CA, Nigg CR, Hill JO, Brink LA, Browning RC. Establishing and evaluating wrist cutpoints for the GENEActiv accelerometer in youth. Med Sci Sports Exerc. 2014; 46:826-33. [PubMed: 24121241] 
Schrack JA, Zipunnikov V, Goldsmith J, Bai J, Simonsick EM, Crainiceanu C, Ferrucci L. Assessing the "physical cliff": detailed quantification of age-related differences in daily patterns of physical activity. J Gerontol A Biol Sci Med Sci. 2014; 69:973-9. [PubMed: 24336819]

Shiroma EJ, Freedson PS, Trost SG, Lee IM. Patterns of accelerometer-assessed sedentary behavior in older women. JAMA. 2013; 310:2562-3. [PubMed: 24346993]

Swartz AM, Strath SJ, Bassett DR Jr, O’brien WL, King GA, Ainsworth BE. Estimation of energy expenditure using CSA accelerometers at hip and wrist sites. Med Sci Sports Exerc. 2000; 32:S450-6. [PubMed: 10993414]

Troiano RP, Berrigan D, Dodd KW, Masse LC, Tilert T, Mcdowell M. Physical activity in the United States measured by accelerometer. Med Sci Sports Exerc. 2008; 40:181-8. [PubMed: 18091006]

Troiano RP, Mcclain JJ, Brychta RJ, Chen KY. Evolution of accelerometer methods for physical activity research. Br J Sports Med. 2014; 48:1019-23. [PubMed: 24782483]

Trost SG, Zheng Y, Wong WK. Machine learning for activity recognition: hip versus wrist data. Physiol Meas. 2014; 35:2183-9. [PubMed: 25340887]

Tucker JM, Welk GJ, Beyler NK. Physical activity in U.S.: adults compliance with the Physical Activity Guidelines for Americans. Am J Prev Med. 2011; 40:454-61. [PubMed: 21406280]

Tudor-Locke C, Barreira TV, Schuna JM Jr. Comparison of step outputs for waist and wrist accelerometer attachment sites. Med Sci Sports Exerc. 2015; 47:839-42. [PubMed: 25121517]

Zhang S, Rowlands AV, Murray P, Hurst TL. Physical activity classification using the GENEA wristworn accelerometer. Med Sci Sports Exerc. 2012; 44:742-8. [PubMed: 21988935] 


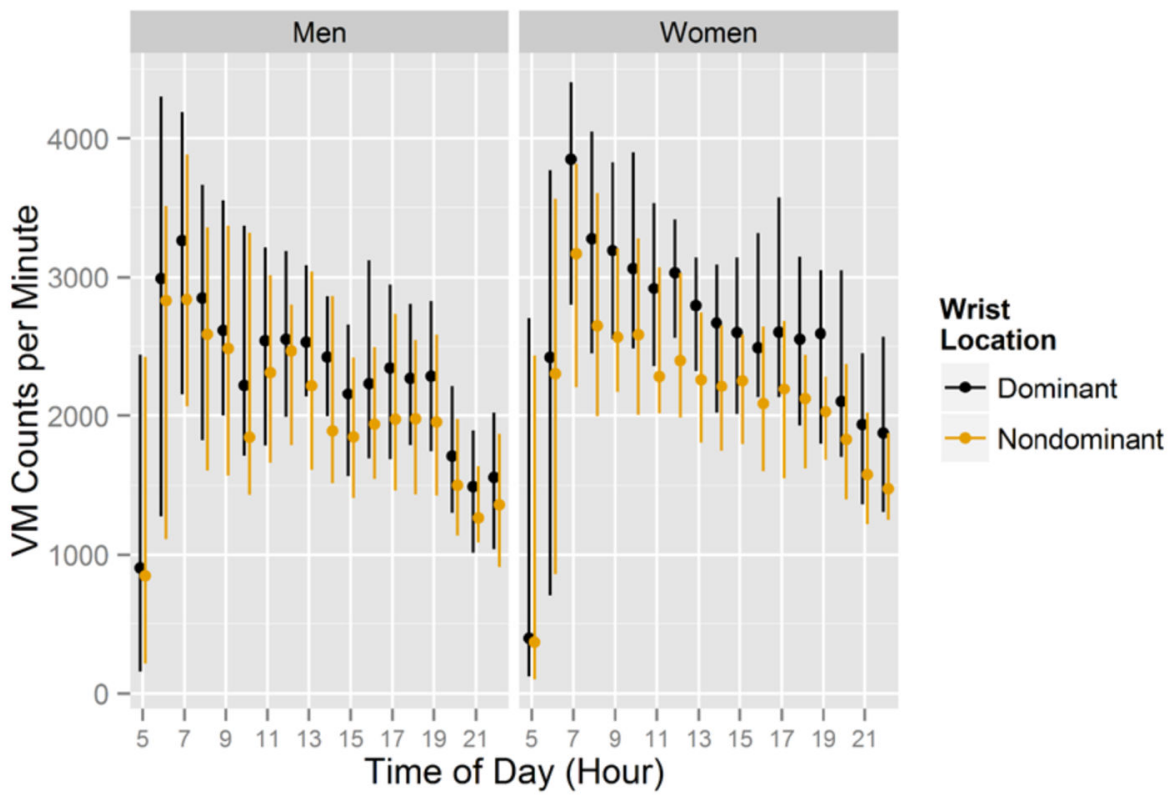

Figure 1.

Physical activity volume throughout the waking day according to wrist accelerometers in men and women, AREA 2012-2013. 


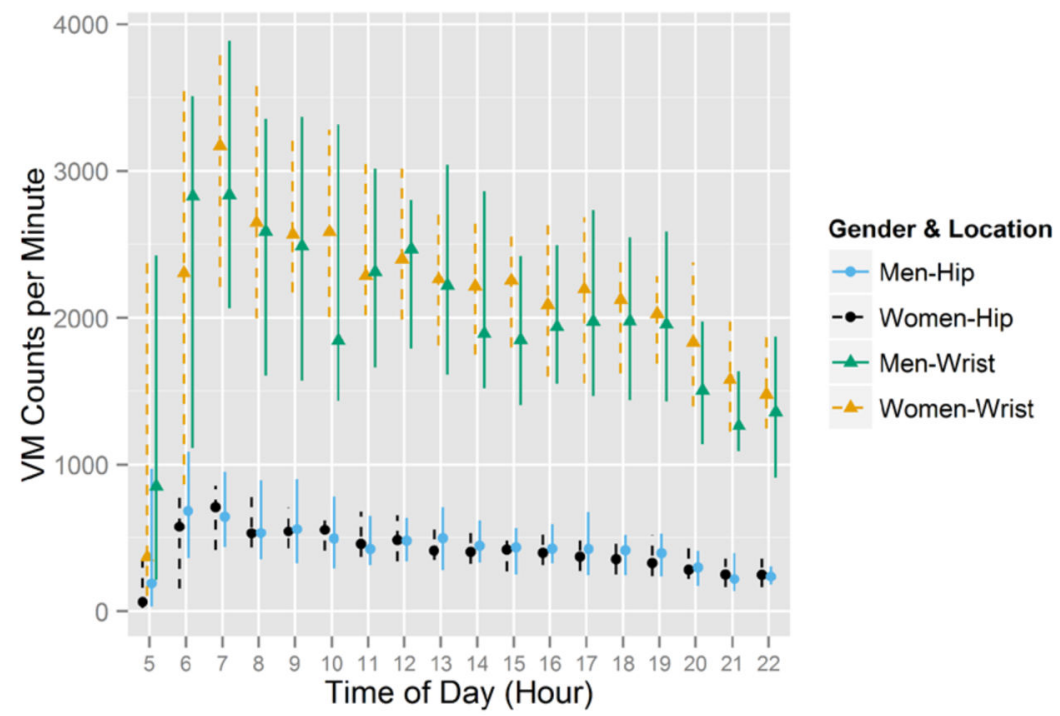

Figure 2.

Physical activity volume throughout the waking day according to hip and nondominant wrist accelerometers in men and women, AREA 2012-2013. 


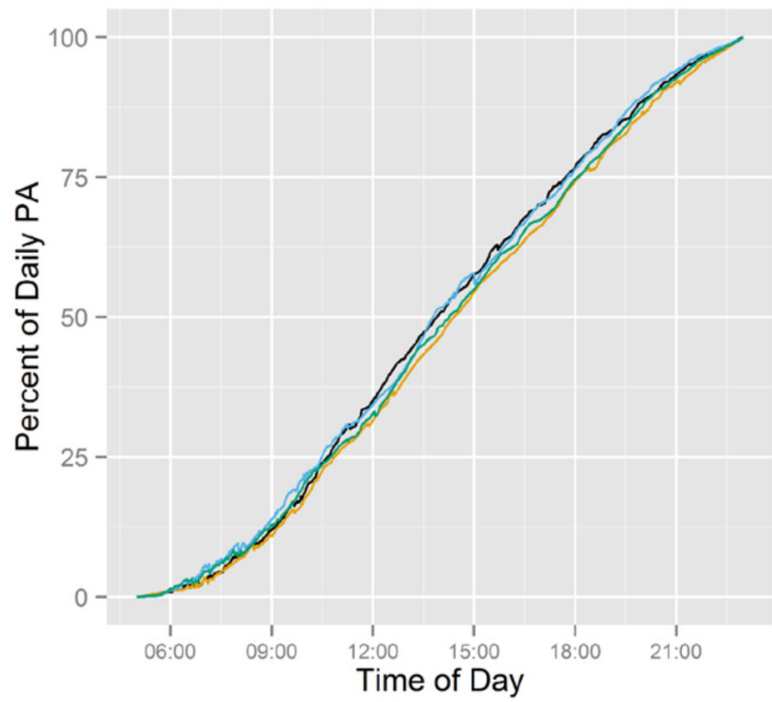

\section{Gender \& Location \\ - Men-Hip \\ - Women-Hip \\ - Men-Wrist \\ - Women-Wrist}

Figure 3.

Cumulative distribution plot of physical activity throughout the day according to hip and nondominant wrist accelerometers in men and women, AREA 2012-2013. 


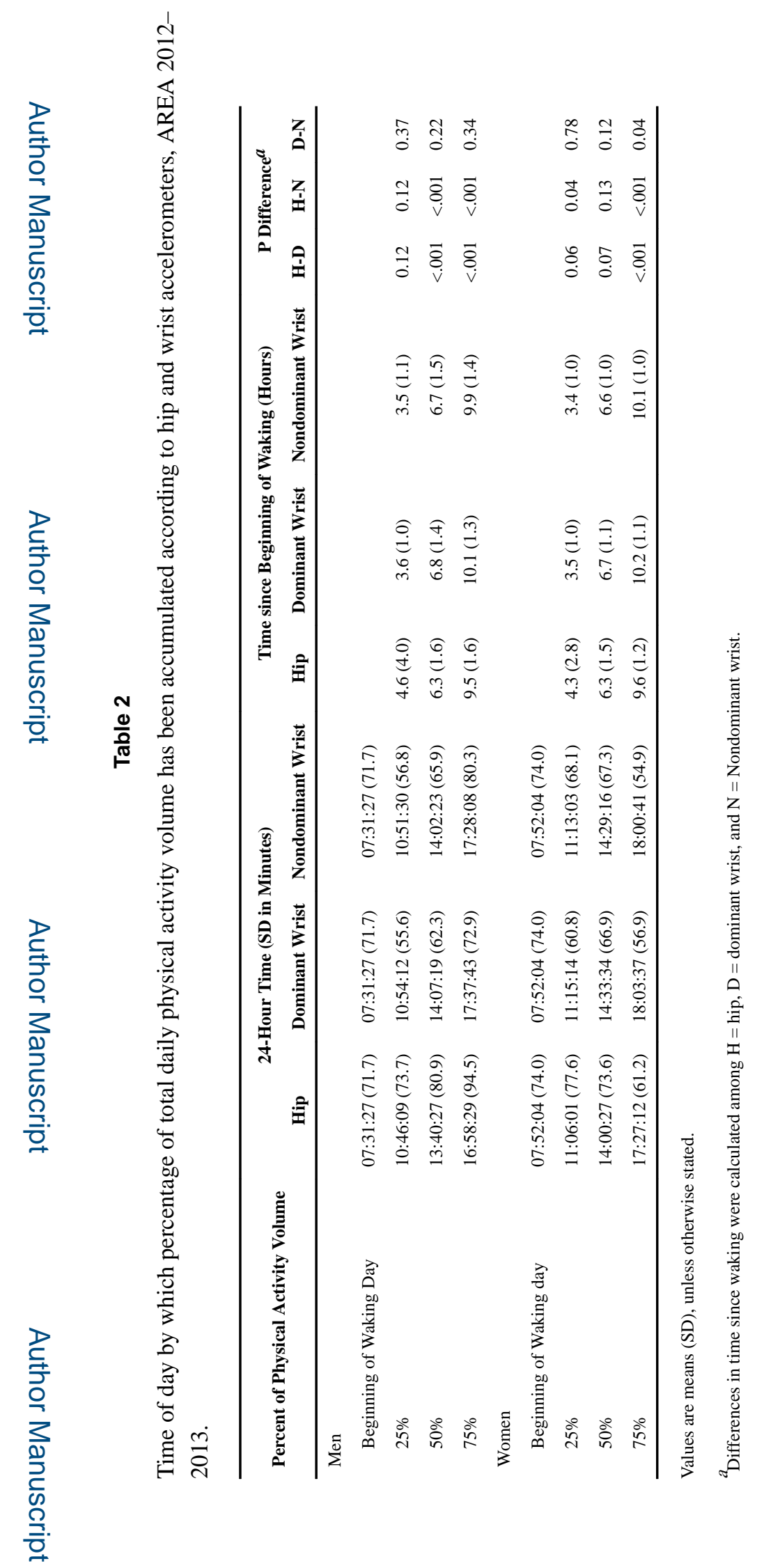

Physiol Meas. Author manuscript; available in PMC 2018 March 21. 